\title{
Fines mobility and distribution in streaming fibre networks: experimental evidence and numerical modeling
}

\author{
Jakob D. Redlinger-Pohn (D) - Melanie Mayr • Gregor Schaub - David Gruber • \\ Stefan Radl
}

Received: 20 April 2020/Accepted: 3 September 2020/Published online: 23 September 2020

(C) The Author(s) 2020

\begin{abstract}
The motion of flocculated fibres in a streaming suspension is governed by the balance of the network strength and hydrodynamic forces. With increasing flow rate through a channel, (1) the network initially occupying all space, (2) is then compressed to the centre, and (3) ultimately dispersed. This classical view neglects fibres-fines: we find that the distribution of these small particles differs in streaming suspensions. While it is known that fibre-fines can escape the fibre network, we find that the distribution of fibrefines is non-homogenous in the network during compression: fibre-fines can be caged and retarded in the streaming fibre network. Hence, the amount of fibre-fines is reduced outside of a fibre network and enriched at the network's interface. Aiming on
\end{abstract}

Electronic supplementary material The online version of this article (https://doi.org/10.1007/s10570-020-03443-9) contains supplementary material, which is available to authorized users.

\footnotetext{
J. D. Redlinger-Pohn $(\bowtie) \cdot$ G. Schaub ·

D. Gruber · S. Radl

Institute of Process and Particle Engineer, Graz University

of Technology, Graz, Austria

e-mail: edu.redlinger@gmail.com

J. D. Redlinger-Pohn

Department of Engineering Mechanics, KTH Royal

Institute of Technology, Stockholm, Sweden

M. Mayr

Institute of Bioproducts and Paper Technology, Graz

University of Technology, Graz, Austria
}

selectively removing fibre-fines from a streaming network by suction, we identify a reduction of the fines removal rate. That documents a hindered mobility of fibre-fines when moving through the network of fibres. Additionally, we found evidence, that the mobility of fibre-fines is dependent on the fibre-fines quality, and is higher for fibrillar fines. Consequently, we suggest that the quality of fibre-fines removed from the suspension can be controlled with the flow regime in the channel. Finally, we present a phenomenological model to compute the length dependent fibre distribution in an arbitary geometry. For a fibre suspension channel flow we are able to predict a length-dependent fibre segregation near the channel's centre. The erosion of a plug of long fibres was however underestimated by our model. Interestingly, our model with parameters fitted to streaming fibre suspension qualitatively agreed with the motion of micro-fibrillated cellulose. This gives hope that devices for handling flocculated fibre suspensions can be designed in the future with greater confidence.

Keywords Fibre segregation - Fractionation · Length-based fibre separation - Hydrodynamic fractionation $\cdot$ Fibre suspension modelling 


\section{Introduction}

"The process of papermaking is one of handling fibre networks and modifying their properties", wrote Sampson (2001) in his review on the characterization of fibre networks. In fact, for most situations, suspensions of fibres, and generally spoken, elongated particles of any kind, is complex. Fibres can segregate and form flocs and networks (of flocs) from elastic interlocking that are transported by the suspending fluid (Soszynski 1987; Bergström 2005; Duffy 2007; Kerekes 2006; Derakhshandeh et al. 2011). The motion of flocculating fibre suspensions depends on the interplay of the network strength and the shearing imposed by fluid motion (Meyer 1964; Schmid and Klingenberg 2000; Schmid et al. 2000). Traditionally, fibre suspensions are regarded as homogeneous mixtures, characterized by the pressure loss (in pipe flow) dependence on the flow rate (for example in Hemström et al. 1976; Norman et al. 1977; Jäsberg and Kataja 2009)). In doing so, a possibly inhomogeneous distribution of fibre-fines, which are defined as particles smaller than $200 \mu \mathrm{m}$ (ISO 16065-2) or particles accepted through a 200 mesh screen $(76 \mu \mathrm{m}$ hole diameter; SCAN-CM 66:05) is neglected. Fibre-fines can be slender fibrils (Steenberg et al. 1960; Mayr et al. 2017a), or fibre fragments and vessel cells with a low aspect ratio $A R$, i.e., a length to diameter ratio of $L_{\text {Fibre }} /$ $D_{\text {Fibre }}<20$.

Only a few early investigations addressed the transport of fibre-fines in a streaming fibre network (Sandgren and Wahren 1960; Steenberg and Wahren 1960). These studies laid the foundation for our still incomplete understanding of spontaneous lengthbased fibre segregation in flow. Also, this almost 60 years old work was not followed up until recently when Redlinger-Pohn et al. (2017ab) utilized the effect of fibre-fines segregation for efficient lengthbased fibre fractionation. The motivation behind this was the push from industry to transform pulp mills into bio-refineries (Mayr et al. 2015). The advantages of Redlinger-Pohn et al.'s "hydrodynamic fractionation" concept are (1) a high selectivity, and (2) no energy loss due to fibre network agitation, i.e. the need to break-up the fibre network as done in classical pressure screening applications (Redlinger-Pohn et al. 2017b, a; Schmid et al. 2019).

In our current contribution we address two issues in the description of length-based fibre segregation: (1) understanding of the quantity and quality of fibre-fines segregation in the wall-normal direction, and (2) the transferability of lab-based understanding to any other geometry and industrial application.

The first objective of this paper is to describe the fibre-fines distribution in a segregated suspension flow: the network of fibres is consolidated to the centre yielding an annular gap that contains primarily fibrefines. We probe streaming networks at flow rates yielding flocculated and dispersed fibre suspension flow. Samples are drawn from different wall-normal positions. That allows us to quantify the segregation of fibres and fibre-fines, as well as a judgment on the fibre-fines' mobility relative to that of the fibres. The methodology follows the previous work of the authors (Redlinger-Pohn et al. 2017a). Additionally, we include in this paper the quality aspect of fibre-fines, i.e., their flake and fibril area following a recent work of Mayr et al. (2017a).

The second objective of our present contribution is the interpretation of the experimental study findings via a phenomenological model that predicts fibre drift (i.e., network consolidation) in a flowing pulp. Specifically, our goal is to predict the local concentration of fibres based on a phenomenological description of the fibre drift speed, i.e., the motion of fibres relative to the suspension. The main driver for our interest in modeling is an improved understanding of the fibre network consolidation in suspension flow.

The structure of this paper is as follows. In "Streaming fibre networks and fines distribution" section we will review relevant literature. We highlight the current understanding of fibre-fines mobility and fibre segregation in suspension flow. We discuss in brief modeling approaches for fiber-transport in literature.

In "Material and methods" we detail the methods and materials. The Hydrodynamic Fractionation Device (HDF) and the sample treatment for the evaluation are described. Details related to the pulp source are given in the Supplementary Material.

In "Experimental results and discussion" section we report the experimental results, starting with the structure of fibre networks and the identification of our flow regimes. That is followed by a detailed discussion of the wall-normal fibre-fines concentration. Last, we present our findings related to the fibre-fines quality.

In "Streaming fibre networks and fines distribution" section we introduce the setup that was studied 
with the complimenting simulation model. The model details are provided in the Supplementary Material. Also, in "Simulation of fibre migration speed and segregation" section we report the simulated fibrefines segregation in dependence on the Reynolds number $\operatorname{Re}$ and fibre concentration on fibre-fines segregation.

In "Conclusion" section we conclude our study and give an outlook on implications and open questions that should be addressed in future studies.

\section{Streaming fibre networks and fines distribution}

A detailed discussion of fibre-fines distribution in streaming fibre networks is scarce. However, a description of the relative motion of fines, specifically organic fibre-fines and inorganic fines such as clay or calcium carbonate particles, can be found elsewhere in cellulose processing: in the field of forming and pressure screening. Forming is the retention of fibres on a screen to generate a network of fibres, specifically a sheet of paper (Parker 1972; Paulapuro 2007). Fines are retained in the established network, which was shown to be a size exclusion process, i.e. fines that are larger than the pore size of the formed network are retained (Athley et al. 2012). The relative motion of fines in the network is hence a function of the fibrefines size and the fibre network's pore structure. Pressure screening is the fibre-length selective acceptance of individualized fibres when flowing through a hole-screen (Karnis 1997). For that, a fibre suspension is agitated to break up the fibre network or flocks. The process description is based on the acceptance probability of fibre-fines (Olson 2001).

\section{Cellulose network suspension flow}

Streaming fibre networks are characterized based on their network state into 5 regimes (shown in Fig. 1) as a function of the fibre concentration and flow rate.

The regimes are reviewed based on early work from Hemström et al. (1976) and Norman et al. (1977) and more recent work from Jäsberg and Kataja (2009) and Haavisto et al. (2017). For increasing flow rate, the regimes are:

(i) Plug flow regime with direct fibre-wall contact The fibre phase forms a continuous

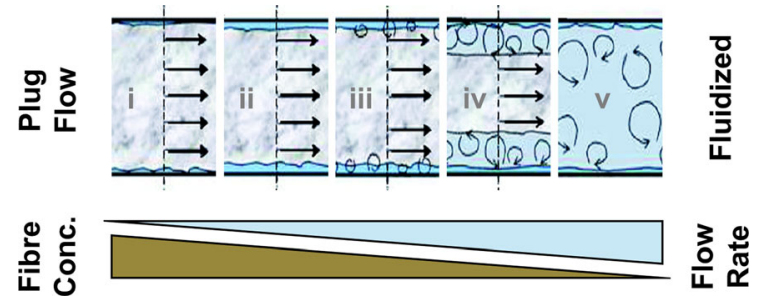

Fig. 1 Fibre suspension flow regimes in dependence on the flow rate (adapted from Jäsberg and Kataja (Jäsberg and Kataja 2009)). The regimes are labeled from $\mathrm{i}$ to $\mathrm{v}$ and range from plug flow to fluidized fibre suspension flow. Turbulent motion is indicated by eddies. The qualitative dependency of the flow regime on the fibre concentration and the flow rate is indicated below

network made from interconnected fibre flocs (Duffy 2006). The network takes the form of the enclosing conduit at low shear. The network's elastic energy pushes the fibres against the wall and this elastic force is balanced by the support force of the wall and a hydrodynamic lift force acting on the fibres.

(ii) Laminar annular plug flow Increased lift force pushes the fibre network from the wall and compresses the network slightly (Meyer 1964). A lubrication layer surrounding the plug forms which is essentially free of fibres but may contain fibre-fines (Steenberg and Wahren 1960). Jäsberg and Kataja (2009) reported the growth of the lubrication layer with the flow rate and Haavisto et al. (2017) determined the fluid viscosity in the gap from resolved velocity measurements. The viscosity within the gap of ca. $0.6 \mathrm{~mm}$ was found constant and ca. twice the fluid viscosity what suggests a small number of fines in their boundary layer of a $0.4 \%$ softwood suspension.

(iii) Plug flow regime with incipient (fluid phase) turbulence The change between regime ii and regime iii is indicated by the onset of turbulence in the lubrication layer. The plug deforms and erodes due to turbulent stress (Nikbakht et al. 2014). Bergström (2005) describes the erosion process on the fibre level. Fibres are torn from the network once the hydrodynamic drag force exceeds the fibre-fibre friction force. 
(iv) Mixed flow regime More fibres are dispersed, and turbulence prevents the formation of a continuous fibre network near the wall. A fibre plug remains at the channel centre.

(v) Fully turbulent flow regime The transition from regime iv to $\mathrm{v}$ is gradual. The diminishing of the fibre plug core cannot be depicted from the pressure loss curve but was recently reported by MacKenzie et al. (2018) using MRI-based velocity measurements. Since MRI technology was not available in our present study, we were unable to distinguish between regime iv and $\mathrm{v}$ in what follows. Based on imaged fibre suspension flow we will describe the suspension flow of the (partially) yielded network in regime iv and $\mathrm{v}$ as channel spanning fibre suspension flow regime.

The relative fibre and fines motion in plug-flow (regime i) can be related to forming, where fine particles move through a fibre network. Its motion in turbulent (or "fluidized") flow (regime v) can be related to pressure screening, where fibres and fibrefines are fluidized and move individually. Laminar annular plug flow (regime ii) was identified to be optimal for high selective fibre-fines separation in our previous study (Redlinger-Pohn et al. 2017a).

\section{Modelling of fibre suspension flow}

Studies of fibre suspension flow on a fibre level and treating the pulp as a continuum were also attempted numerically. Most studies on the fibre level focus on the simpler case of dilute suspension with noninteracting fibres, for example Krochak et al. (2009), Marchioli et al. (2010), Redlinger-Pohn et al. (2016). Only a few discuss the suspension flow of flocculating and networking fibre suspensions. Pioneering was the work by the group of Klingenberg (Schmid and Klingenberg 2000; Schmid et al. 2000; Switzer and Klingenberg 2004). A milestone is a work of Lindström and Uesaka (2008) who simulated industrial paper forming considering polydispersity, i.e. a fibre ensemble with a distribution of fibre length and fibre diameter. Unfortunately, fibre-fines, i.e. particles smaller than $200 \mu \mathrm{m}$, were neglected by them as their large number would have resulted into a strongly increased computation cost.
Continuum level simulations are computationally much cheaper and hence attracted our interest. However, this affordability of a simulation typically comes at the cost of precision, i.e., the pulp must be assumed as one continuum and different classes as fibres and fibre-fines are not resolved (examples for such models include rheology based models, e.g., Hammarström 2004; Cotas et al. 2017)). Models balancing precision and computational effort can be found in particleladen flows: Brennan (2001), for example, describes the size-dependent particle motion as a size-dependent drift flux. For the mixture phase, i.e., water and fibres, rheological models representing the suspension's flow behavior were applied. Krochak et al. (2010) presented another approach of fibre modelling in the Eulerian frame. They calculated the fibre orientationdependent viscosity for a dilute and a semi-dilute system, i.e., $1<n L_{\text {Fibre }}<A R$, where $n$ is the number of fibres per unit volume and $A R$ the fibre aspect ratio. $A R$ in their comperative experiment was 50. Such systems are easily dispersable (Martinez et al. 2001), which does not reflect our experimental conditions.

\section{Material and methods}

Fibre pulp

Three types of pulps (SW, L100, S100), and two mixtures (L90S10, L50S50) were used in our present study. A summary of the most important properties, determined with an L\&W Fibretester + (ABB, Lorentzen \& Wettre, Sweden), are listed in Table 1. SW and $L$ are softwood pulps from Sappi (Sappi Gratkorn, Austria) and Zellstoff Pöls (Zellstoff Pöls, Austria), respectively. S is hardwood pulp form Sappi (Sappi Gratkorn, Austria). The number state the mass-based mixing ratio. More details related to the used fibre pulps are given in the supplementary material.

$L_{1}$ and $L_{3}$ are the length-weighted and volumeweighted mean fibre length, respectively. $A R_{O}$ is the arithmetic mean aspect ratio for a given length class. The coarseness $c s$ is the line density of the fibres. The fibre network is characterized by the crowding number $\mathrm{N}_{\mathrm{CW}}$, a metric to quantify the interaction of fibres and their networking state, defined on basis of the mass concentration $C$ (Kerekes and Schell 1992, 1995; Celzard et al. 2009) as: 
Table 1 Summary of key pulp properties (Characteristic length-weighted mean fibre length $L_{1}$, and volume-weighted mean length $L_{3}$, as well as fibre coarseness cs. $A R_{O}$ is the arithmetic mean aspect ratio. Resulting crowding number $\mathrm{N}_{\mathrm{CW}}$ for fibre concentration in the pulp $C_{\text {Feed }}$ of $0.1 \%$ and $0.2 \%$ )

\begin{tabular}{|c|c|c|c|c|c|}
\hline & SW & L100 & L90S10 & L50S50 & S100 \\
\hline Length-weighted fibre Length $L_{1}[\mathrm{~mm}]$ & 1.76 & 2.19 & 1.79 & 1.11 & 0.84 \\
\hline Volume-weighted fibre Length $L_{3}[\mathrm{~mm}]$ & 2.41 & 2.48 & 2.25 & 1.55 & 0.85 \\
\hline$A R_{O}(0.03<L<0.2 \mathrm{~mm}$, fines $)[-]$ & 5.0 & 4.8 & 4.5 & 3.9 & 3.6 \\
\hline$A R_{O}(0.2 \mathrm{~mm}<L<1 \mathrm{~mm}$, fibre $)[-]$ & 17.1 & 19.6 & 27.3 & 34.4 & 35.9 \\
\hline$A R_{O}(1 \mathrm{~mm}<\mathrm{L}$, fibre $)[-]$ & 74.8 & 73.0 & 70.9 & 65.9 & 63.4 \\
\hline Fibre coarseness cs $[\mathrm{mg} / \mathrm{m}]$ & 0.17 & 0.16 & 0.14 & 0.08 & - \\
\hline Crowding number $\mathrm{N}_{\mathrm{CW}}, C_{\text {Feed }} 0.1 \%$ & 8.9 & 14.8 & 11.5 & 7.4 & - \\
\hline Crowding number $\mathrm{N}_{\mathrm{CW}}, C_{\text {Feed }} 0.2 \%$ & 17.8 & - & - & - & - \\
\hline
\end{tabular}

$\mathrm{N}_{\mathrm{CW}}=5\left[\frac{k g}{m^{3}}\right] C[\%] \frac{L_{1}^{2}}{c s}$

Fibre-fibre interactions are expected for $\mathrm{N}_{\mathrm{CW}}>1$, fibre connectivity for $\mathrm{N}_{\mathrm{CW}}>16$ with in average 2 to 3 contract points per fibre, and rigidity for $\mathrm{N}_{\mathrm{CW}}>60$ with in average more than 3 contact points per fibre (Kerekes and Schell 1992; Martinez et al. 2001; Celzard et al. 2009). The pulp in our study was prepared to result in moderate fibre crowding numbers, i.e. $\mathrm{N}_{\mathrm{CW}} \approx 16$, which is known as gel-crowding number. For this condition, we expect fibre-fines motion relative to the network motion, i.e. in the lateral direction to the streaming direction (Martinez et al. 2001).

\section{Hydrodynamic fractionation device (HDF)}

The experimental method of this study is identical to the method reported in Redlinger-Pohn et al. (2017a). The channel height $H$ and the channel width $W$ were $15 \mathrm{~mm}$ and $3 \mathrm{~mm}$, respectively. $W$ was chosen small for optical accessibility in the previous study. Samples were drawn from the suspension via a side-channel (to which suction was applied) at a position $52 \mathrm{H}$ downstream of the inlet (Fig. 2a).

The sampling height in the wall-normal direction is dictated by the suction flow rate, termed accept flow rate $\phi^{+}$here (see white dashed line in Fig. 2c). The fibre network interface was determined from averaging recorded images (details are available in the Supplementary Material). The interface position for a fibre network depends on the hydrodynamic stress,

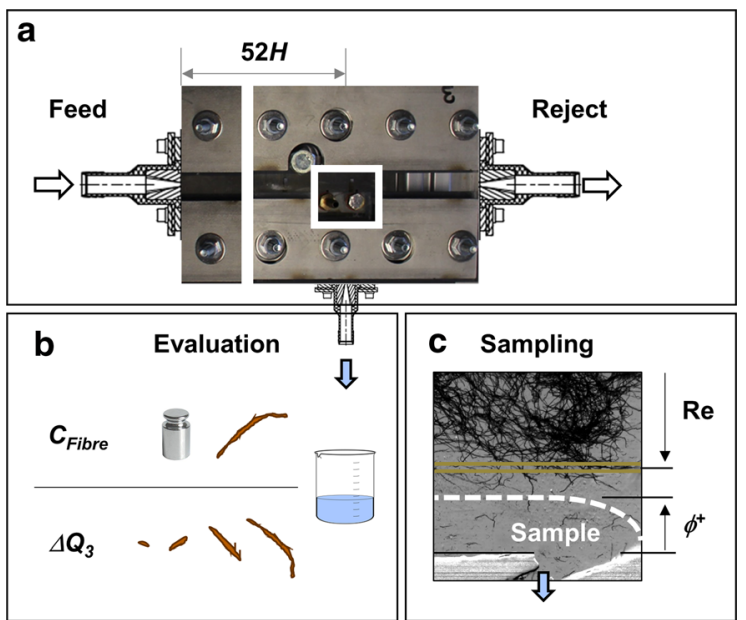

Fig. 2 Illustration of the fibre suspension sampling in the Hydrodynamic Fractionation Device HDF. a laboratory HDF design with the sampling position $52 \mathrm{H}$ downstream the inlet. The detail highlighted is the observation window presented with a snapshot in c. b Evaluation procedure: the fibre concentration $C_{\text {Fibre }}$ and length distribution $\Delta Q_{3}$ is measured from the collected sample. c Snapshot of the streaming suspension. The interface of segregated fibres is denoted by the double-line and is sensitive to the Reynolds number $R e$. The sampling depth increases with the relative sample volume $\phi^{+}$

characterized in what follows by the Reynolds number Re. Re was computed as a guiding parameter based on the mean pulp velocity (calculated from the flow rate and channel cross-section), the channel hydrodynamic diameter and the kinematic viscosity of water.

The evaluation procedure is illustrated in Fig. 2b: The solid concentration $C$ was determined gravimetrically after drying at $105{ }^{\circ} \mathrm{C}$. The fibre-length distribution $\Delta Q_{3}$ was measured with an L\&W 
Fibretester + . From these we calculate the mass shift of material into the sample, expressed by the mass fraction $m_{s}$ and the selectivity $s$ :

$m_{s}=\frac{C_{\text {Sample }}}{C_{\text {Feed }}}$,

$s=\frac{\Delta Q_{3, \text { Sample }}(0.1 \mathrm{~mm})}{\Delta Q_{3, \text { Feed }}(0.1 \mathrm{~mm})}$.

The mass fraction $m_{s}$ quanitifies the transport of solid mass into the accept. The sample is depleted of solid material for $m_{s}<1$. The selectivity $s$ compares the relative volume-based amount of the class of fibrefines in the sample to that in the feed. $s$ hence quantities a preferred transport of fibre-fines $(s>1)$ or fibres $(s<1)$ into the sample. The fibre-fines class was defined including particles with a length from 0 to $0.2 \mathrm{~mm}$, hence a class mean of $0.1 \mathrm{~mm}$ expressed by $\Delta Q_{3}(0.1 \mathrm{~mm})$. The product $m_{s} s$ states the relative fibre-fines concentration $C_{\text {fibre-fines }}$ in the sample as compared to the feed. Multiplying that with the relative sample volume $\phi^{+}$:

$\phi^{+}=\frac{\dot{V}_{\text {Sample }}}{\dot{V}_{\text {Feed }}}$,

quantifies the absolut suspended fibre-fines material in the sample as compared to the feed. We denote the relative fibre-fines removal, expressed by the fines fraction, as $f$ :

$f=\phi^{+} m_{s} s=\frac{\dot{V}_{\text {Sample }}}{\dot{V}_{\text {Feed }}} \frac{C_{\text {Sample }}}{C_{\text {Feed }}} \frac{\Delta Q_{3, \text { Sample }}(0.1 \mathrm{~mm})}{\Delta Q_{3, \text { Feed }}(0.1 \mathrm{~mm})}$.

$f$ is explained on hand of the snapshot presented in Fig. 2c: the white dashed line demarcates the relative sample volume $\phi^{+}$upstream the sampling position. The fibre-fines quantified by $f$ are suspended in the sample flow, i.e. below the white dashed line. $f$ compares the measured amount of fibre-fines in the sample to the theoretical amount of fibre-fines for the case that those are homogeneously distributed in the channel. That is the case for $f=\phi^{+} . f<\phi^{+}$expresses a depletion, for example by fibre-fines captured in the fibre network. $f$ quantifies the local fibre-fines fraction at the sampling positions in wall-normal direction and hence the fibre-fines distribution.

\section{Experimental results and discussion}

Before we discuss the fibre-fines distribution in the streaming fibre network, we identify the flow regime. Illustrations of the network in one half of the channel are shown in Fig. 3. The fibre network interface determined from image averaging is indicated as double line. The sampling positions are indicated by white arrows.

At Re 1300 (Fig. 3a) fibres segregated towards the channel centre in a plug which thickness was approximately $0.6 H$. For that the local $\mathrm{N}_{\mathrm{CW} \text {, local }}$ increases to approximately 15 . The fibre-depleted gap between the wall and the fibre network had a thickness of $0.2 H$. The gap height was found from observing a region that was essentially free of fibres. The regime is identified as regime ii: laminar annular plug flow. At Re 2500 (Fig. 3b) the fibre network expanded what yielded a gap with a smaller thickness as that in regime ii, and
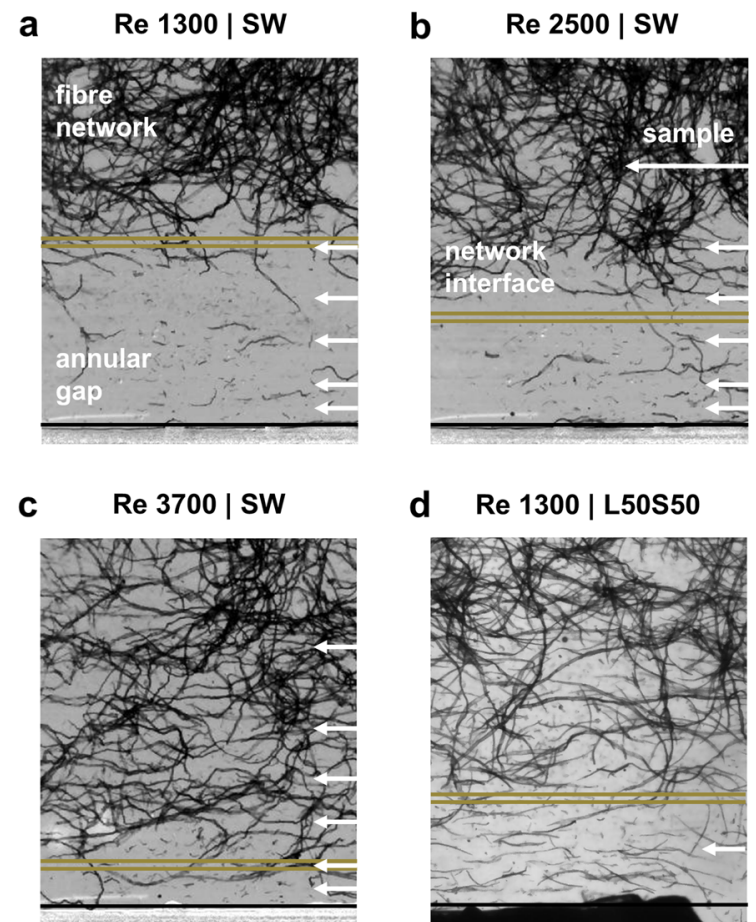

Fig. 3 Illustrations of the investigated flow regimes. The white golden double line indicated the network interface which was identified based on changes in the mean grey value of the image sequence (Redlinger-Pohn et al. 2017a). The white arrows denote the sampling depth. The suspension below was sampled for analysis. a-c snapshots for SW sampled at increasing wallnormal distance. $\mathrm{d}$ is a snapshot for mixture L50S50. The feed fibre concentration $C_{\text {Bulk }}=0.1 \%$ 
containing dislodged fibres. The case correlates to regime iii: turbulent annular plug flow. At Re 3700 (Fig. 3c), the network of fibres spans the channel cross-section and the fibre motion can be regarded agitated, i.e. dispersed fibre suspension flow. The degree of fluidization, i.e. core fluidized or not, could not be determined. The case correlates to regime iv to regime v. Figure $3 \mathrm{~d}$ presents the flow situation of L50S50, i.e. pulp mixture with a large content of medium-sized fibres in comparison (Table 1). The near-wall region of the suspension is populated with shorter fibres and no fibre-free gap is present. The fibres at the channel core appear comparable to what is seen for softwood only (Fig. 3a-c), whilst the fibres closer to the wall appear less entangled and shorter. An interesting observation as it could indicate segregation of softwood and hardwood fibres form the mixture in channel flow. A more thorough discussion of that is however not in the scope of this paper which focuses on the mobility of fibre-fines.

Fibre-fines distribution across the channel

Figure 4 presents the fibre-fines fraction $f$ sampled from the streaming networks versus the relative sample volume $\phi^{+} . \phi^{+}$correlates to sampling positions in the wall-normal direction. The network interface determined from averaging recorded images of the streaming suspension [(Redlinger-Pohn et al. 2017a), and Supplementary Material] is indicated by a double line in Fig. 4a. The region of networked and/or connected fibres is indicated by the bar in Fig. 4b-d.

Figure 4a compares the fibre-fines fraction $f$ of the three different flow cases to the theoretical homogenous distribution, i.e. a line with a slope of unity indicated in red. Apparent is, that none of the measured fraction falls on top of the homogenous line. At $\operatorname{Re} 2500$ between $\phi^{+}=0.15$ to 0.2 , the increase in fines fraction is, however, steeper than 1 , i.e., the slope is larger than unity. Re 2500 fines fraction $f$ appears identical to that observed for $\mathrm{Re}$ 1300 for small $\phi^{+}$, and to Re 3700 for high $\phi^{+}$. The variation of the sampled fibre-fines fraction with the Reynolds number is best understood comparing the trend with $\operatorname{Re}$ at $\phi^{+}=0.15$ in Fig. 4 a to the corresponding state of flow in Fig. 3a-c. The fibre-fines are homogeneously distributed across the channel for an agitated state of fibre suspension flow, whilst the number of fibre-fines near the wall is smaller for networks compressed to the channel center. The fibrefines distribution for the 3 states of flow is presented individually in Fig. 4b-d. Data for $f$ which appeared to follow a linear trend were fitted with a line (slope $k$ and intersect $d$ are stated in the figure). These linear fits are represented as solid lines for the fitted range, and as dashed lines for the range outside.

It appears that for Re 1300 (Fig. 4b) we mostly sampled the gap suspension. The largest probing depth was just at the network interface. For that region, we measured a linear increase of $f$ with $\phi^{+}$at an inclination of approximately 0.5 . That means, that only half of the fibre-fines of a homogenous mixture were suspended within the gap.

For Re 3700 (Fig. 4d) we were able to sample deep into the channel spanning fibre suspension. The increase of $f$ with $\phi^{+}$is linear and close to 1 with a small negative offset. Here, we can only speculate on the interpretation of this offset. We hypothesise, that a very thin near-wall region that corresponds to $\phi^{+}$of 0.01 (approximately $0.15 \mathrm{~mm}$ of sampling depth) is depleted of fibre-fines. The offset is however an extrapolation, and the nature of the offset may result from an interaction of fibres with fibre-fines in the agitated state during sampling. Haavisto et al. (2017) resolved the near-wall fluid structures by optical coherence tomography measurements. We suggest to include resolved flow measurement in a future study alongside sampling to study the near-wall fibre-fines distribution in agitated fibre suspension flow. A regime, that may be found in industrial suspension transportation. The inclination of $f$ for $\operatorname{Re} 3700$ decreases for $\phi^{+}>0.2$. A local decrease of the fibre-fines concentration in the agitated network, and consequently a strong increase outside our measurement range at $\phi^{+}>0.3$, respectively the channel centre appears odd and unphysical. The decrease in inclination is interpreted as fibre-fibre interaction. Fibres sampled from deep in the channel have a higher chance to interact with other fibres what impacts their motion and may hinder their removal. In turn, that may impact the sampling flow and consequently a change in the measured fibre-fines fraction. The important finding is a nearly homogenous distribution of fibrefines in the near-wall area at $\operatorname{Re} 3700$. It is reasonable to assume the fibre-fines distributed homogeneously also at larger distances from the wall.

Re 2500 (Fig. 4c) shows a richer behaviour and 3 distinct slopes of $f$ versus $\phi^{+}$were identified. The 

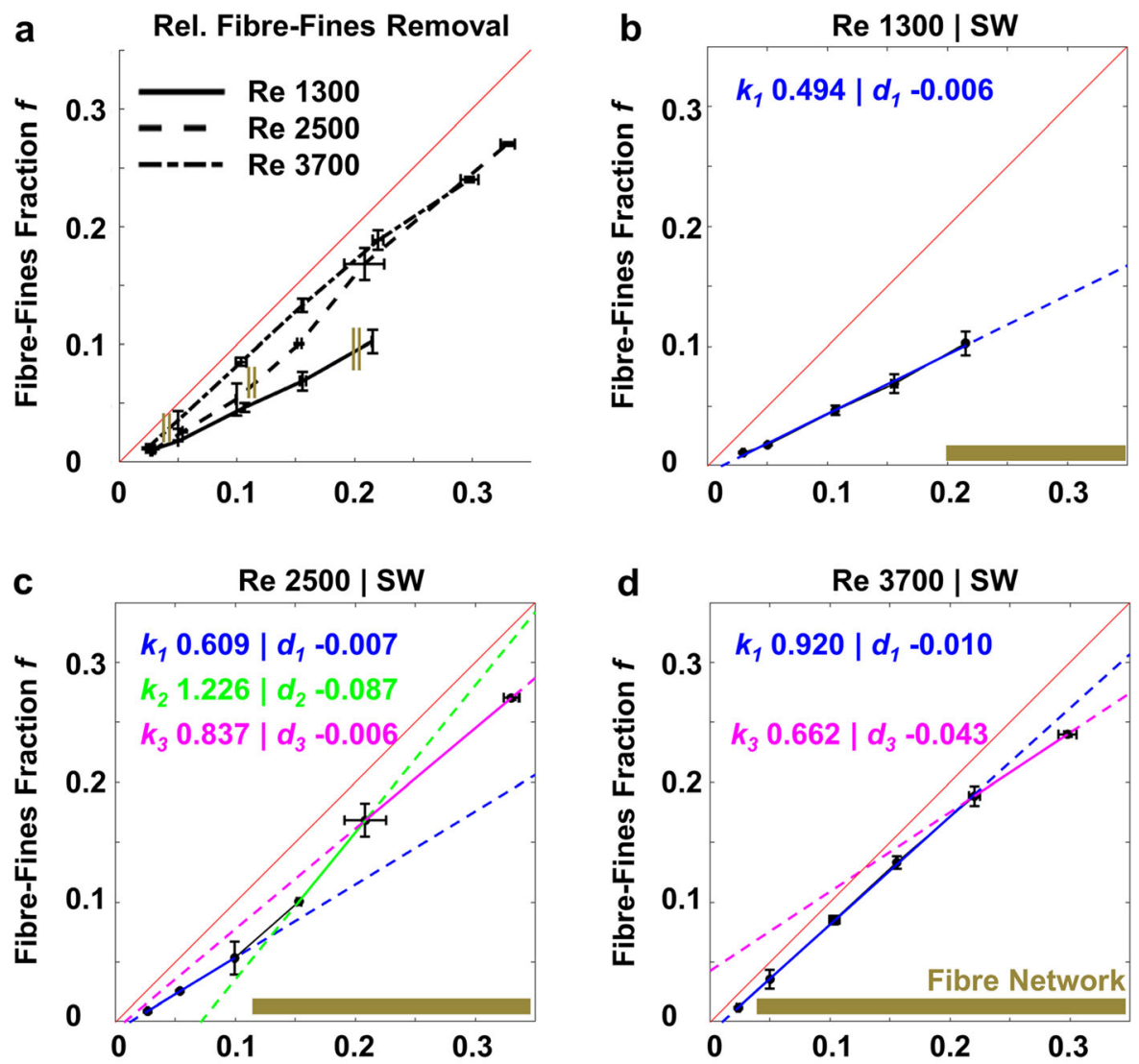

Rel. Sample Volume $\phi^{+}$

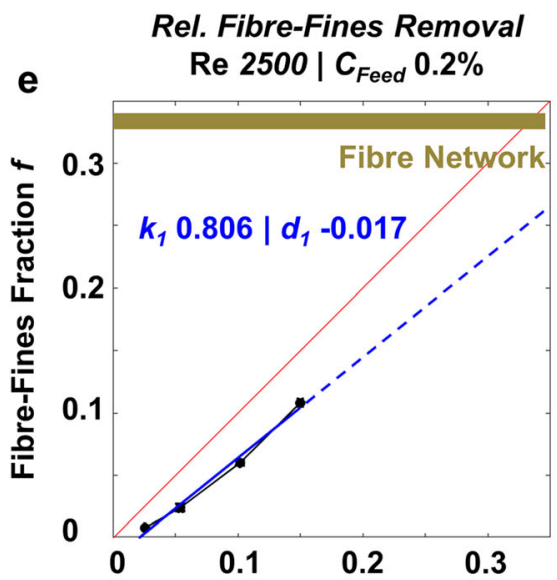

Rel. Sample Volume $\phi^{+}$

Fig. 4 Relative fibre-fines fraction $f$ over the relative sample volume $\phi^{+}$of SW. Panel a-d illustrate data for $\mathrm{N}_{\mathrm{CW}}=9$, and panel e data for $N_{C W}=18$. a: results comparison of $\operatorname{Re} 1300$ (solid), Re 2500 (dashed), and Re 3700 (dash-dotted) to the theoretical case of homogenous distribution (solid red). The

double line indicated the network interface per case. $\mathbf{b}-\mathbf{d}$ are the detailed results per flow situation, i.e. Re. The results are fitted by lines which parameters are stated in the figure. $k$ is the slope, and $d$ is the intercept offset. The golden bar indicates the region of segregated and networked fibres 
sample positions at low $\phi^{+}$were outside the network in the gap region. The inclination was 0.6 (blue line) and shows a depletion of fibre-fines that is comparable to the situation characterized by Re 1300 (Fig. 4b). Probing inside the fibre network, the slope of $f$ first increased to approximately 1.3 what states a local increase in the fibre-fines fraction and then leveled to approximately 0.8 for higher $\phi^{+}$. The latter is comparable to Re 3700 (Fig. 4d) and, as argued for that case, a homogenous distribution of fibre-fines in the network core at the bulk concentration can be assumed. We checked for the plausibility of the measured fibre-fines fraction inside and outside the fibre network the mass conservation. The intersection points of the individual segments of the linear fits in Fig. $4 \mathrm{c}, \phi^{+}{ }_{1,2}$ and $\phi^{+}{ }_{2,3}$, calculate to 0.13 and 0.21 , respectively. $\phi^{+}{ }_{1,2}$ corresponds to the documented network height from averaged images of approximately 0.12 (see Supplementary Material). The concentration, i.e. the slope for the $3^{\text {rd }}$ segment from $\phi^{+}$of 0.21 to 0.3 was assumed as bulk concentration for what $k_{3}$ is set to 1 in the mass balance. The cumulative amount of fibre-fines up to $\phi^{+}=0.3$ calculates as, mass $_{\text {Fibre-Fines }}=\int_{0}^{\phi_{1,2}^{+}} k_{1} d \phi+\int_{\phi_{1,2}^{+}}^{\phi_{2,3}^{+}} k_{2} d \phi+\int_{\phi_{2,3}^{+}}^{0.3} 1 d \phi$,

to 0.27 . That agrees well with the cumulative fibrefines fraction for a homogenous distribution which is $\phi^{+}$and hence 0.3 , with a relative error of $6.5 \%$.

In summary, we find from the sampled fibre-fines fraction a (1) depletion of fibre-fines outside the network, speculated to result from fibre-fines interaction with the fibre network, (2) a consequent accumulation of fibre-fines confined to the network interface, and (3) a homogenous distribution of fibre-fines at bulk concentration in the network core. The accumulation of fibres-fines at the network interface indicates a hindered fibre-fines transport through the network pores: fibre-fines may be caged and retained as in a filter that is formed by intersecting fibres [comparable to the mechanical retention argument of fines in formed fibre networks (Athley et al. 2012)]. A consequence is, that the mobility of fibre-fines in the streaming network will depend on the network structure.

The fibre networks at $C_{\text {Feed }}=0.1 \%$ and $\mathrm{N}_{\mathrm{CW}} \sim 9$ deformed in the tested Re range. A minimum flow rate was needed in the device to avoid a fibre accumulation at the channel inlet. The hypothesis of hindered fibre- fines transport in a streaming network at plug flow conditions was hence tested at higher concentration. $\operatorname{Re} 2500, C_{\text {Feed }}=0.2 \%$ and $\mathrm{N}_{\mathrm{CW}} \sim 18$ (Fig. 4e) presents a flow case comparable to plug flow (regime i). The fibre-fines are removed from a coherent network of non-dispersed fibres that stretch across the channel. The increase of $f$ with $\phi^{+}$is linear with a slope of approximately 0.8 up to $\phi^{+}=0.15$. Indeed, the reduced amount of sampled fibre-fines from the fibre network indicates a hindered fibre-fines transport and a filtering effect. That supports our hypothesis, that fibre-fines can be caged in streaming networks.

\section{Fibre-fines distribution in streaming networks of softwood and hardwood pulp mixtures}

In the second set of experiments, we fixed the sampling height, respectively sampling flow rate to $\phi^{+}=0.06$. That corresponds to a sampling position of $0.9 \mathrm{~mm}$ from the wall, which is large for the fibre-fines but small for the longer fibres. The fibre-fines fraction $f$ and the selectivity $s$ for 3 pulps, softwood (L100), and two softwood-hardwood mixtures (L90S10 and L50S50) are presented and compared in Fig. 5. The sampling depth is comparable to the second sampling point $\left(\phi^{+}=0.05\right)$ from the wall in SW (Fig. 3, second arrow from the wall). SW results for $\mathrm{Re}=1300$ (Fig. 4b) and $\mathrm{Re}=3700$ (Fig. 4d) are drawn in Fig. 5 for comparison.

Overall the fibre-fines fraction $f$ for the pulp mix is largely independent on the mixture and comparable to what was measured for SW (Fig. 5a). Especially in the annular plug flow regime (i.e., at Re 1000) and the dispersed fibre-suspension flow regime (i.e., at $R e$ 4000). Only in the transition range ( $\operatorname{Re} 2000$ and $\operatorname{Re}$ 3000) we note an increase of $f$ at lower Re for L50S50 compared to L90S10 and L100 what is addressed to the easier dispersion of the weaker network (the corresponding crowding numbers $\mathrm{N}_{\mathrm{CW}}$ for the cases were $7.4,11.5$ and 14.8, respectively). In particular, we found for $\operatorname{Re} 4000$ the sampled fibre-fines fraction $f \approx 0.055$, what is in the range of the sample volume, i.e. $\phi^{+}=0.06$. The selectivity $s$ (Fig. 5b) is low and close to 1 for the dispersed case at $\operatorname{Re}=4000$. Fibres and fiber-fines were sampled alike.

For $\operatorname{Re} 1000$ the sampled fibre-fines fraction $f \approx 0.02$. The selectivity $s$ was dependent on the pulp mixture and decreased with an increasing amount of short-fibre hardwood pulp. In the observation of the 

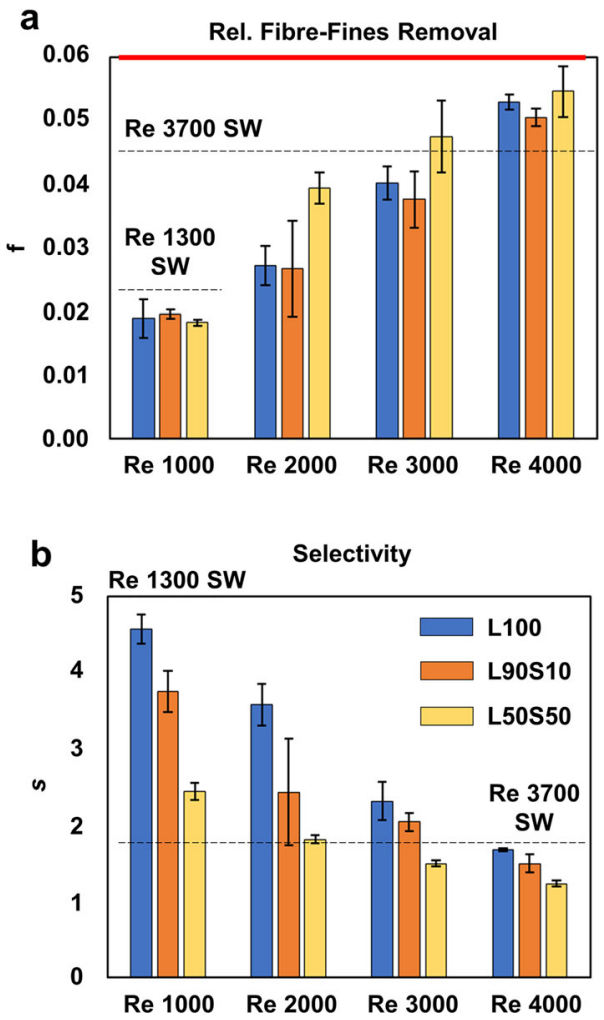

Fig. 5 Results and simple standard deviation for constant sampling and changing pulp properties, i.e. mixtures of softwood and hardwood pulp: L100, L90S10, and L50S50. a Fibre-fines fraction $f$, $\mathbf{b}$ selectivity $s$ corresponding to data presented in panel a. The corresponding value for SW is drawn as a dashed line. The red line at $f=0.06$ represents the theoretical sample of homogenously distributed fibre-fines

suspension flow (Fig. 3d) it appeared, that longer fibres formed a network and shorter fibres were dispersed in the vicinity of the wall. A partial segregation of smaller fibres which were removed by sampling. Consequently, the selective $s$ was lower.

For the intermediate Re number, i.e. Re 2000 and Re 3000, the sampled fibre-fines fraction $f$ increases progressively for softwood L100 and softwood with a small amount of hardwood L90S10 whilst the selectivity $s$ decreases. A different qualitative behaviour was noticed for L50S50. The sampled fibre-fines fraction $f$ increased comparable strong for Re 2000 and only moderate thereafter. The selectivity $s$ was ca. 2 and low compared to L100 and L90S10. As stated before, the network of the pulp with a crowding number $\mathrm{N}_{\mathrm{CW}}=7.4$ is weak and consequently easier to disperse. More fibre-fines are then to be found near the wall and within the probing depth at lower Re.

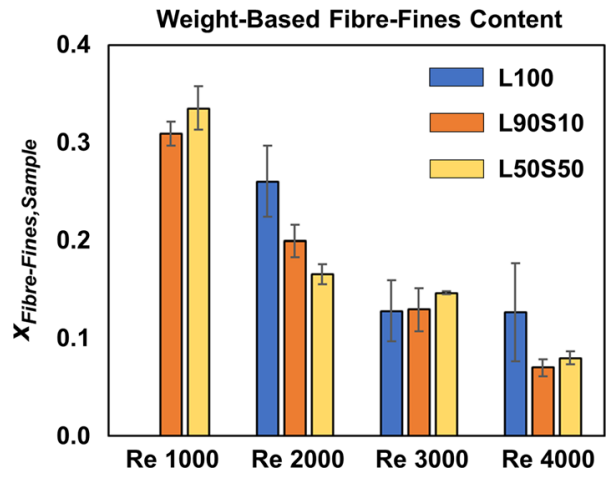

Fig. 6 Fibre-fines fraction $x_{\text {Fibre-Fines,Sample }}$ in the sample depending on the flow situation, i.e. Re and pulp mixture

The samples from the streaming pulp mixtures were also fractionated with a 200-mesh screen in a Britt Jar Drainage device. The mass of the fine and coarse fraction was determined after drying at $105{ }^{\circ} \mathrm{C}$ what allowed the evaluation of the fines fraction in the sample, $x_{\text {Fibre-Fines,Sample }}$. The results are presented in Fig. 6.

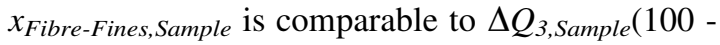
$\mu \mathrm{m}$ ), and is hence correlated to the selectivity $s$ (see Eq. (3)). The difference in the methods is, that the selectivity is based on optical detection of fibres which could not resolve particles $<3.5 \mu \mathrm{m}$ (and we set the evaluation length threshold to $33 \mu \mathrm{m}$ ), for example, fibrils and finest particles. Those are captured in the mass-based determination of the fibre-fines content. The method relies on the exclusion of particles smaller than $75 \mu \mathrm{m}$, what includes acceptance of high aspect fibres/fibrils longer than $200 \mu \mathrm{m}$ (a detailed comparison of fibre-fines methods including weight-based and optical methods is for example given by Hyll (2015)). Qualitatively, the trends agree: the fibre-fines content in the sample decreases with $\mathrm{Re}$ and the mixture fraction of hardwood (Fig. 6). For comparison, we normalize the results of $x_{\text {Fibre-Fines,Sample, and }}$ $s$ for every pulp mixture with their value at Re 4000 . The results are presented in Fig. 7 and show differences between the gravimetrically and optically determined change in fibre-fines fraction. For both, we see a trend towards higher fibre-fines contribution at lower Re. The trend is however larger for the gravimetrically determined fraction $x_{\text {Fibre-Fines,Sample, }}$ and more notoriously for L90S10 and L50S50. Reasoning is provided in the discussion on the qualitative aspects of the sampled fibre-fines. 


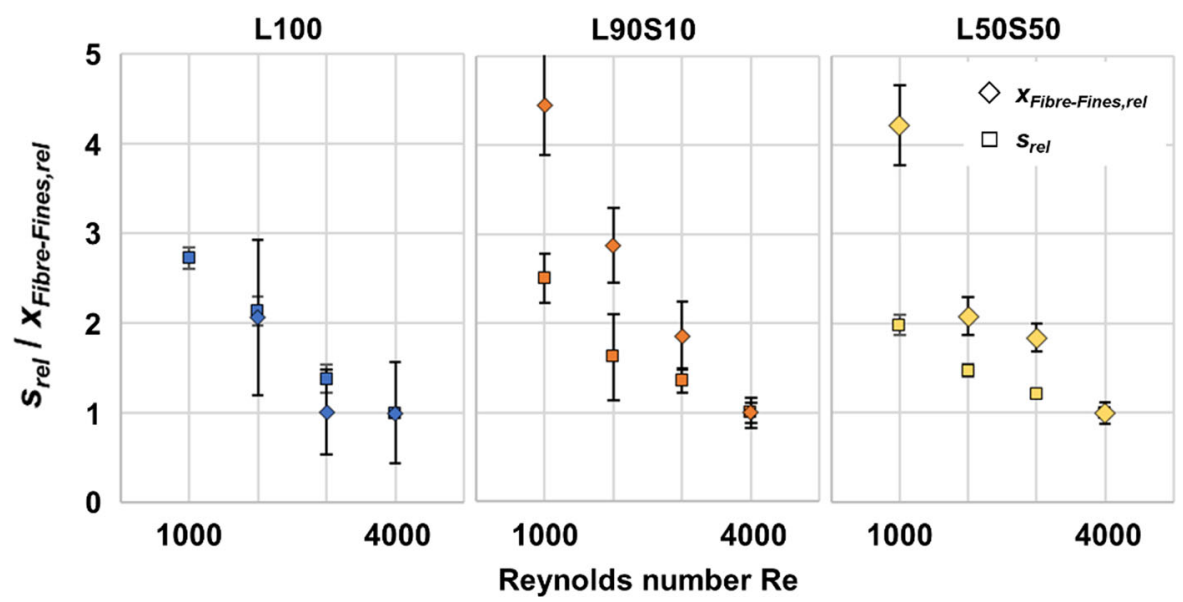

Fig. 7 Selectivity $s$ (squares) and gravimetrical fibre-fines content $x_{\text {Fibre-Fines }}$ (diamonds) per pulp mixture normalized by the value at $\mathrm{Re}=4000$ and their simple standard deviation. The

We speculate that the discrepancy between the two methods results from the contribution of fibrillar material, i.e. crill as reported for example by Steenberg et al. (1960). This fibrillar material cannot be captured by the L\&W Fibretester + (Mayr et al. 2017a). It is conceivable, that these slender fibrils escape the network of fibres more easily for what their relative contribution increases. A detailed analysis of the number and relative contribution of fibrillar fibrefines in the sample could not be performed. However, we will discuss in the next section the use of microscopic methods that allow the quantification of fibrils (the microscopic method mostly resolves coarse fibril bundles).

Qualitative aspects of the sampled fibre-fines

Last, we will take a closer look at the qualitative aspect of the sampled fibre-fines which could be deduced from the L\&W Fibretester + measurements. The length-averaged mean length and the arithmetic mean aspect ratio were calculated. The upper length limit was set to $200 \mu \mathrm{m}$. Additionally, we determined the relative fibrillar area of L100 and L50S50 samples microscopically (Mayr et al. 2017a). Given the small sample amount that was collected for low Re cases, measurements were only feasible for $\operatorname{Re} 3000$ and $\mathrm{Re}$ 4000 cases.

We found the mean fibre length in the sample independent on Re resulting to approximately $110 \mu \mathrm{m}$ for pure softwood pulp (SW and L100). The mean results illustrate the relative increase in fibre-fines content for decreasing $R e$ and hence increasing fibre segregation and compare the two measurement methods

fibre length was approximately $105 \mu \mathrm{m}$ for $10 \%$ hardwood content (L90S10), and approximately $95 \mu \mathrm{m}$ for $50 \%$ hardwood mixture (L50S50). Similar values were found for the fibre-fines fraction (i.e. $<$ $200 \mu \mathrm{m})$ in the pulp. The ratio of the mean fibre length of the accept to the feed is between 0.98 to 1 .

The arithmetic mean aspect-ratio $A R_{O}$ of fibre-fines in the samples was also independent on Re with values between 4.5 and 5.0 for softwood pulp (SW and L100). The mean aspect ratio decreased to approximately 4.3 to 4.5 for $10 \%$ hardwood content (L90S10), and 3.8 to 3.9 for $50 \%$ hardwood content (L50S50). Similar values were found for the fibre-fines fraction in the pulp (see Table 1). The larger aspect ratios were between 16 and 19 in the feed. Those particles can be regarded stiff and would not form a network on their own (Kerekes and Schell 1992) but may be caged by the network of larger fibres. The case of SW at $C_{\text {Feed }}=0.2 \%$ at $\operatorname{Re} 2500$ differed in the sense that fibre-fines were actively filtered from the network and not segregated from the network as it happens during the formation of a water gap. The aspect ratio of the detectable fibre-fines was comparable.

The fibrillar content for the analyzed samples and the feed pulp is summarized in Table 2. Interestingly, the fibre-fines fibrillar area in the sample exceeds the fibrillar area in the feed. That agrees with the earlier finding and speculation of fibrillar fines escaping the network more easily and hence accumulating in the accept. The consequence is a change of the fibre-fines quality from separation in streaming networks. 
Table 2 Relative area of the fibre fibrils measured microscopically employing the method described by Mayr et al. (Mayr et al. 2017a). The samples were taken at $\phi^{+} \approx 0.06$

\begin{tabular}{llll}
\hline Pulp & \multicolumn{2}{l}{ Relative fibrils area } & Feed \\
\cline { 2 - 4 } & Samples $\operatorname{Re} 3000$ & Samples $\operatorname{Re} 4000$ & $0.583 \pm 0.004$ \\
\hline L100 & $0.704 \pm 0.001$ & $0.701 \pm 0.020$ & $0.536 \pm 0.010$ \\
L50S50 & $0.605 \pm 0.035$ & $0.615 \pm 0.005$ & $0.639 \pm 0.155$ \\
S100 & & & \\
\hline
\end{tabular}

Apparent is also the initial higher fibril area for hardwood (S100) compared to softwood (L100). Consequently, the amount of fibrillar fines in the sample is higher for mixtures with hardwood for what we found a stronger increase of $x_{\text {Fibre-Fines, rel }}$ compared to $s_{\text {rel }}$. Interesting is that the trend of a moderate addition of hardwood (L90S10) and an equal addition of hardwood (L50S50) to softwood pulp was similar. The reason for that appears unclear to us at this point. We suggest to follow-up the investigation of fibrefines quality separation with dedicated experiments that set the levels of non-fibrilar (also known as primary) and fibrillar (also known as secondary) fines. For example, cellulose fibre pulp can be prepared as in Mayr et al. (2017b); fibre-fines can be removed by screening and a known amount of primary and secondary fines can be added to the pulp for suspension flow testing.

\section{Simulation of fibre migration speed and segregation}

In laboratory experiments, we quantified the fibre length profile in the wall-normal distance via extracting a wall near layer of suspension. To quantify this effect in industrial scale equipment is much more difficult. The strategy is hence to describe the principle of fibre and fibre-fines segregation in streaming suspensions via a mathematical model that is scale independent. Specifically, we undertook an attempt to implement a phenomenological continuum model into the widely-used open-source simulation program OpenFOAM ${ }^{\circledR} \quad$ (OpenFOAM Foundation). We adopted a continuum model for total mass and momentum equation (i.e., the Navier-Stokes equations with a spatially variable viscosity), in addition to a mass balance of fibres (see the details in the next paragraph). This is in contrast to previous work (Schmid and Klingenberg 2000; Switzer and Klingenberg 2004) that aimed at tracking individual fibres using a discrete approach, as well as previous work (Krochak et al. 2010) that aimed at tracking the particle orientation distribution in regimes characterized by $N_{C W}<8$. In our approach we consider nonBrownian fibres suspended in water in a dense regime (i.e., crowding numbers sufficiently larger than unity are allowed). The model neglects an orientation preference of the fibres, which is accurate for the channel center but a heuristic assumption for the fibre depleted area near the wall. Our approach enables a fast evaluation of the model and the study of large systems, i.e. equipment at industrial scale.

Effectively, a differential single fibre-species mass balance equations is solved with a phenomenological approach for the fibre-suspension relative drift velocity (essentially we consider a force balance between network-induced and hydrodynamic forces, see the "Appendix" for details). The key novelty in our modeling approach is, that we compute the local fibre concentration and do not assume the concentration profile ad hoc as done in previous work (Cotas et al. 2015, 2017). Also, we account for the elasticity of the fibre network. Plastic deformation, i.e. fibre plug erosion, at higher Re (Nikbakht et al. 2014) is, however, not captured by our present model. The idea is to propose a phenomenological model, and parameterize it based on the results of Cotas et al. $(2015,2017)$. The model is described in detail in the "Appendix" and has the current limitation that the fibre length is assumed to be mono-disperse. Model fitting and validation is documented in the Supplementary Material (the fitted model parameters, if not stated otherwise, were $K=10^{-7} \mathrm{~m}^{2}$ corresponding to a fibre with a length of $2 \mathrm{~mm}, K_{e l}=10^{7} 1 / \mathrm{s}$ for the elastic network response, and $n_{c, E}=2$ ). In what 
follows we present and discuss results for a $2 \mathrm{D}$ slice and a 3D channel that corresponds to our experiment (Fig. 2a). All these simulations were performed until a steady state was reached. Results of a rigorous grid convergency study are available in the Supplementary Material (see Chapter 2.2). The results of our 3D simulations presented next were run on the "base case" computational mesh that featured 200, 25, and 10 cells in the $x$-, $y$-, and $z$-direction, respectively ( $x$ is the main flow direction; resolutions in $y$ - and $z$ direction are for the the half width and height of the channel). 2D simulation results were generated on a computational mesh that featured 200, and 25 cells in the $x$-, and $y$-direction, respectively (resolutions in the $y$-direction is for the the half width of the channel).

Model predictions for different flow speeds

In Fig. 8 we present the concentration profile normalized by the bulk concentration $C_{B u l k}=0.1 \%$ of a $2 \mathrm{D}$ slice for two flow rates corresponding to $\mathrm{Re}=1300$ (Fig. 8a) and $\operatorname{Re}=3700$ (Fig. 8b).

The network interface is sharp for $R e=1300$ and diffuse for $R e=3700$. For the higher Re, the network consolidation to the channel centre (i.e. $y / H=0$ ) is less pronounced and not developed until $x / H=53.3$ what corresponds to $0.8 \mathrm{~m}$. There are interesting

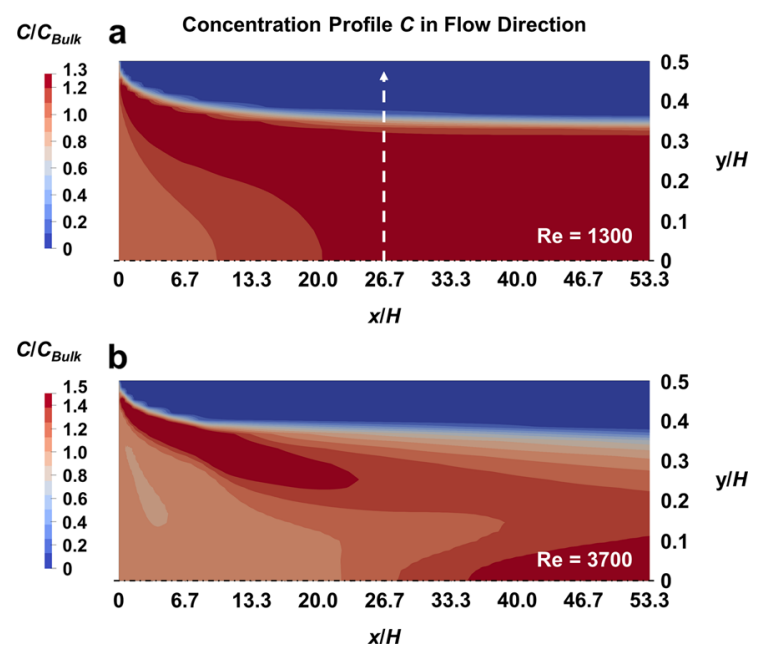

Fig. 8 Distribution of the normalized fibre concentration $C$ for, $\mathrm{a}: \operatorname{Re}=1300$ and, $\mathrm{b}: \operatorname{Re}=3700$ ( $y=0$ is the channel center). The figure is compressed in the x-direction. Feed concentration $C_{\text {Bulk }}=0.1 \%$, model parameters corresponding to a fibre length of $2 \mathrm{~mm}$ ). The white-dashed arrow denotes the sampling line of results presented in Fig. 9 concentration maxima near the inlet up to approximately $x / H=20$ and from approximately 35 . The annular gap region is predicted free of fibres (in this case of length $2 \mathrm{~mm}$ ) for low and high Re.

Subsequent comparison of the concentration and velocity (quantified by $\mathrm{Re}$ ) is done at $x / H=26.7$ as indicated in Fig. 8a. Figure 9a, b presents normalized concentration and velocity profiles for $C_{B u l k}=0.1 \%$ and Fig. $9 \mathrm{c}, \mathrm{d}$ for $C_{B u l k}=0.2 \%$.

At $C_{B u l k}=0.1 \%$, our model predicts that an increasing flow speed decreases the size of the network-wall gap. Also, a dramatic change of the velocity profile is noticed when going from $R e=1300$ to $\operatorname{Re}=2500$ (see Fig. 9b). Specifically, the interface of the plug (located at $y / H=0.35$ for $\operatorname{Re}=1300$ as observable in Fig. 9a) erodes with increasing flow speed (see the concentration and velocities profiles for $\operatorname{Re}=2500 \ldots 7400$ in Fig. 9a and 9b, respectively). Interestingly, the velocity profiles for $\operatorname{Re}=2500 \ldots$ 7400 in Fig. 9b suggests the formation of a thin wallnear layer at $y / H>0.47$ featuring an extremely high velocity gradient. However, the plug does not fully disperse up to $\operatorname{Re}=7400$, as is noticeable from the respective concentration profile in Fig. 9a, and hence the model predicts no fibres in this high velocity gradient layer. Our current model does not include plastic deformation which would capture plug erosion. Interesting is hence the decreasing gap thickness with an increasing Re from 1300 to 2500 (i.e. from $0.2 H$ to $0.12 \mathrm{H}$ in the experiment and $0.15 \mathrm{H}$ to $0.1 \mathrm{H}$ in the simulation, see Fig. 9a). As the parameter for the elastic network response is unchanged a smaller gap implicates a more relaxed fibre network with increasing Re. Nikbakht et al. (Nikbakht et al. 2014) measured the fibre network size as function of Reynolds stresses and identified three regions (1) linear decrease during network consolidation at low $\mathrm{Re}$, (2) a nonmonotonic behaviour at intermediate Re and (3) plug erosion at higher $\mathrm{Re}$, when turbulent stresses exceeded the network yield-stress. The results from our phenomenological model do not allow us to comment on the transition mechanism of network suspension flow. They, however, invite to readdress the transition in a dedicated study including elastic and plastic network deformation.

The trend at higher concentration, $C_{B u l k}=0.2 \%$, is comparable: plug dispersion and consequently a change in the flow profile (see Fig. 9c, d) is noticed with increasing Re. The normalized concentration and 
$\longrightarrow \operatorname{Re}=1300-\operatorname{Re}=2500---\operatorname{Re}=3700 \quad--\operatorname{Re}=7400$

Concentration Profile $C$
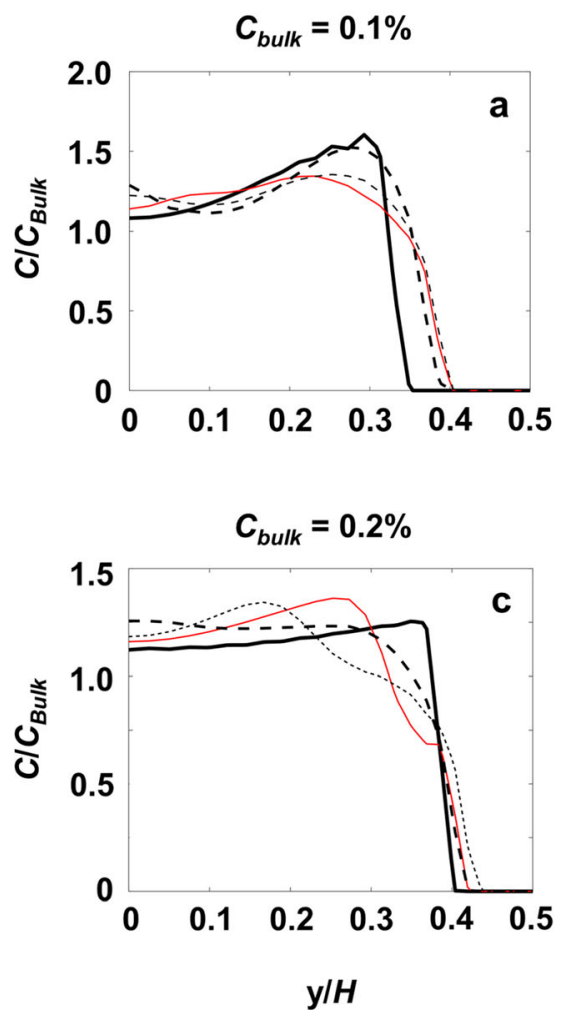

Fig. 9 Distribution of normalized fibre concentration $C / C_{B u l k}$ (a, c) and normalized velocity $U / U_{B u l k}(\mathrm{~b}, \mathrm{~d})$ across the channel at $x / H=26.7$ (white dashed line in Fig. 8a). Feed concentration

the size of the gap is smaller for $C_{B u l k}=0.2 \%$, as the network is stronger at higher concentration. The formation of a thin wall-near layer at $y / H>0.47$ featuring an extremely high velocity gradient is also observed for $C_{\text {Bulk }}=0.2 \%$ and $\mathrm{Re}=2500 \ldots 7400$. Thus, we conclude that the strong effect of the Reynolds number on the fibre dispersion and velocity profile is present for both studied concentrations.

In Fig. 10 we present the simulation results of a quarter of a full 3D channel corresponding to our experimental case of $H=15 \mathrm{~mm}$ and $W / H=0.2$ (i.e., $W=3 \mathrm{~mm})$. The suspension flow is evaluated for a length of $x / H=26.7$ (i.e., $x=0.4 \mathrm{~m}$ ) downstream the inlet what corresponds to the sampling position in the 2D case (see Fig. 8a, white dashed line). Compared to the 2D case (Fig. 8) a somewhat more diffusive fibre distribution is noticed for the $3 \mathrm{D}$ case. Shear also introduces fibre migration in the spanwise direction

\section{Velocity Profile $U$}
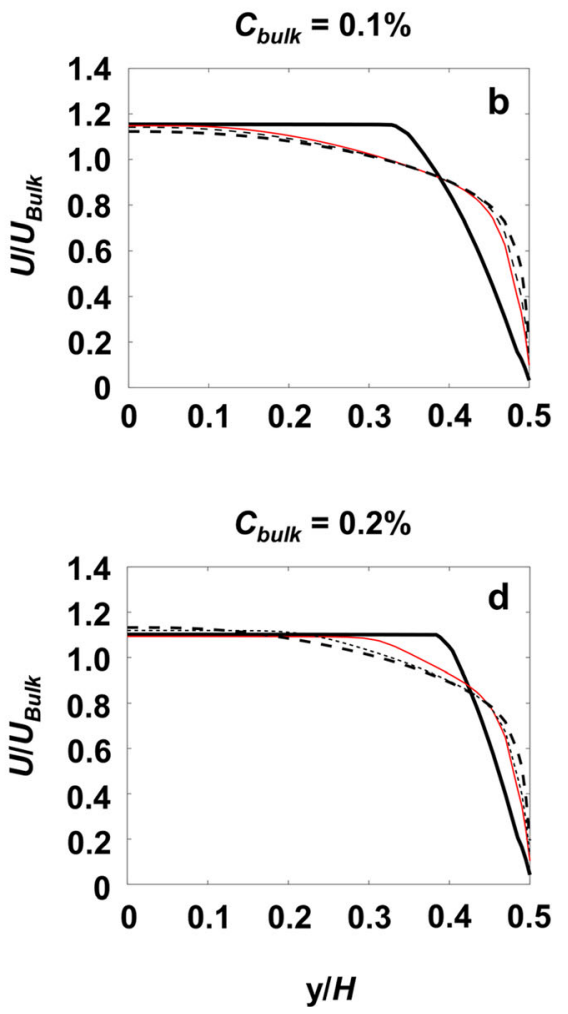

$C_{\text {Bulk }}=0.1 \%(\mathbf{a}, \mathbf{b})$ and $C_{\text {Bulk }}=0.2 \%(\mathbf{c}, \mathbf{d})$. For all simulations, a fibre length of $2 \mathrm{~mm}$ was adopted

(i.e., the direction of the channel width $W$ ), leading to a substantially higher fibre concentration in the fibre plug compared to the 2D case. Thus, higher fibre concentrations are expected for the presented model in $3 \mathrm{D}$ compared to $2 \mathrm{D}$, simply because there is an additional particle-free layer forming in the z-direction. Such a high concentration was not observable in the experiment for which the plug coarsening was discussed based on the idea of an uni-axial compression (in the shear direction, i.e., the direction of the channel height $H$ ) only. In fact, the plug is compressed bi-axially and the increase in fibre concentration and network density is even stronger from the consolidation of the fibre network towards the channel centre.

The accumulation of fibres in the channel corner (see Fig. 10) is noticed as a model error associated with (i) the initialization of the flow field (initially, the concentration in the channel was assumed to be $C_{B u l k}$; 


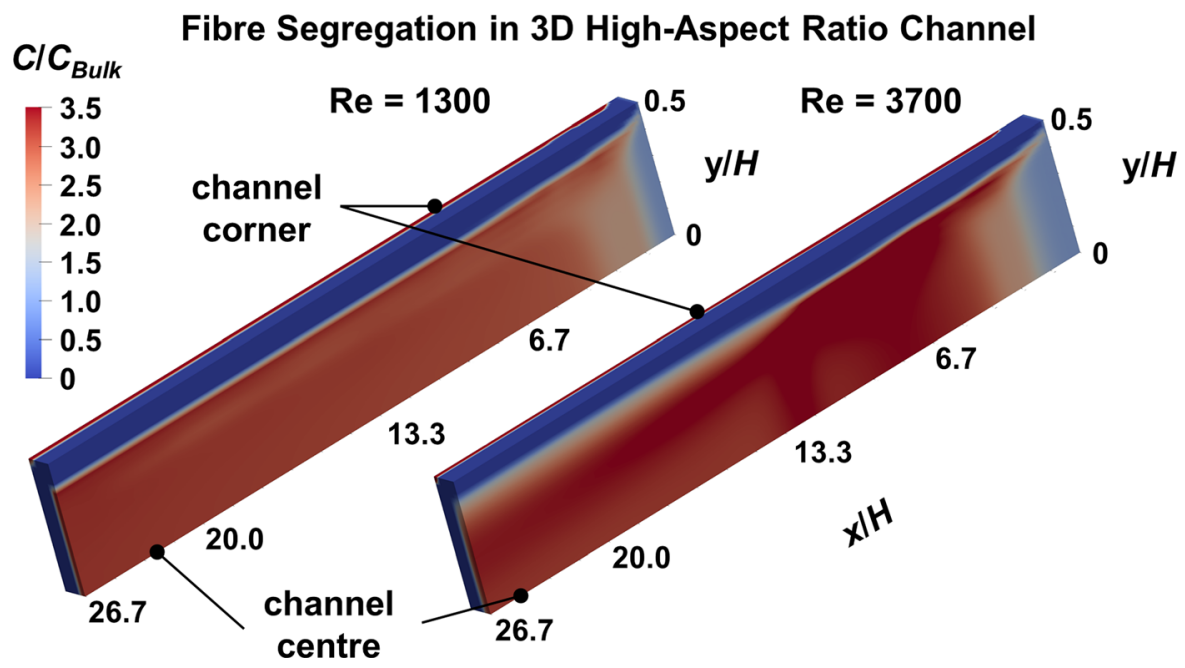

Fig. 10 Normalized fibre concentration distribution for low Re (left panel) and high Re (right panel) shown in one quarter of the full channel. $y=0, z=0$ is at the channel centre; walls are at $y /$

additional simulations that started from a channel initially void of fibres showed no accumulation of fibres in the channel corner), as well as (ii) limitation due to adopting the continuum assumption: low stress in the channel corner is not inducing migration which expresses reality. However, for interaction on the fibre level, fibres from the corner would be pulled by fibres more in the center what causes migration in reality. That is relevant for simulations of geometries with stagnant zones, for example, channel flow, but irrelevant for the simulation of, for example, pipe flow.

Model predictions for different fibre lengths

Last we present in Fig. 11 simulation results for different fibre lengths, expressed by variations of the parameter $K$. From the base value $K=10^{-7} \mathrm{~m}^{2}$ we reduced it by two orders of magnitudes what corresponds to the same relative reduction in fibre length, i.e. from $2 \mathrm{~mm}$ to $20 \mu \mathrm{m}$. The reader should consider that we assume an identical inlet fibre concentration of $C_{\text {Bulk }}=0.1 \%$, as a result of our model limitation to mono-disperse suspension.

Decreasing $K$ (i.e., smaller fibres) leads to a significantly thinner size of the annular gap region that is depleted of fibres. Noteworthy is the difference of near-wall fines depletion for $K=10^{-9} \mathrm{~m}^{2}$. Our model considers migration as fibre response to differences in the network stress. Hence, also fines are
$H=-0.5 \ldots 0.5$ and $z / H=-0.1 \ldots 0.1$; the $z$-(i.e., spanwise) direction is not indicated for clarity). $C_{B u l k}=0.1 \%, 2 \mathrm{~mm}$ fibre length

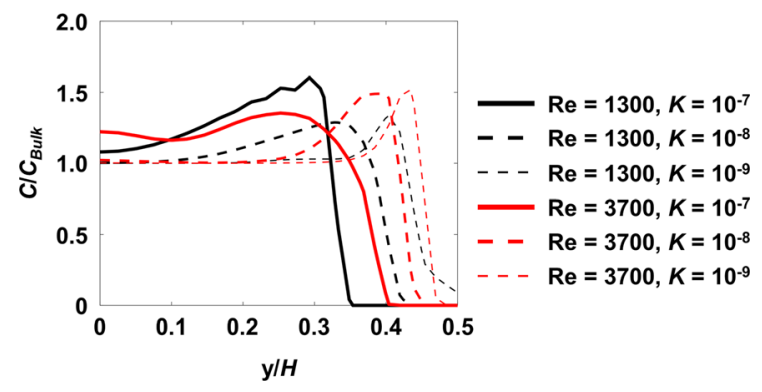

Fig. 11 Normalized fibre concentration across the channel height $H\left(y=0\right.$ is the channel centre). $C_{B u l k}=0.1 \%, K_{e l}$ $=10^{7} 1 / \mathrm{s}$ and $n_{c, E}=2 . K$ (i.e., the fibre length parameter) and Re are varied

represented as a network and not as non-flocculating particles which is lesser the case for flake like fibrefines but may represent entangled micro-fibrillated cellulose (MFC). Fibre-fibre and fibre-wall interaction is not resolved in our model. The fibre depletion near the wall in our model is hence not from polevaulting. Interestingly, a near-wall depletion of fibres, i.e. lower concentration, with fibres present at the wall was recently reported for micro-fibrillated cellulose (MFC) by Koponen et al. (2019). They calculated the wall-concentration from optical coherence tomography OCT velocity measurements and pressure loss data to be approximately $0.2 C_{\text {Bulk }}$ to $0.25 C_{\text {Bulk }}$. The wall-concentration for our case $K=10^{-7} \mathrm{~m}^{2}$ and $\mathrm{Re}=1300$ is approximately $0.1 C_{B u l k}$, calculated with 
a model fitted to entangled fibre pulp suspension flow (Cotas et al. 2015, 2017) and scaled to represent fibrefines. We want to highlight those findings in qualitative similarity worthy to be revisited in future studies.

Last, it is worth discussing the effect of Re on the concentration profiles shown in Fig. 11: as can be seen, increasing Re leads to a reduction of the gap size. It is interesting to note that our model predicts that for shorter fibres this change is smaller (i.e., the change in the fibre free near-wall layer is less) compared to longer fibres. Thus, an increase of Re has a smaller effect on short fibre distribution compared to longer ones, mainly because the former are already better dispersed across the channel.

\section{Summary of simulation results}

In annular plug flow, fibre migration is driven by differences in the network stress, i.e., we assumed migration along the divergence of the network stress tensor. This "driving force" is proportional to the projected area of a fibre, i.e. its length times the diameter. The elasticity of the fibre network must be considered when modeling the fibre migration speed. Modeling of turbulent stresses is also of key importance as it decreases the mean shear near the plug's interface and hence reduces the fibre migration speed. The model is aimed for performance, and this is why we have decided to not trace individual fibres. Hence, the plug erosion at higher $\mathrm{Re}$ is not captured. Considering fibre network elasticity, we document a network dispersion with increasing Re. That finding suggests that fibre network elasticity may contribute to the transition in fibre suspension flow from laminar to turbulent. We suggest, that better understanding of the contribution of elastic network deformation and erosion from a comparative study including experiments with fibres of different stiffness, i.e. glass fibres, carbon fibres, and dedicated simulations.

Albeit, the model was fitted to entangled fibrenetwork flow, a reduction of the projected area parameter $K$, i.e. representing fibre-fines in the $\mu \mathrm{m}$ range, qualitatively captured the suspension flow of MFC. Not captured by the continuum model so far is the fibre-fibre interaction and the fines enrichment in the fibre network interface. From the experimental results, we argued, that the fines enrichment results from a filtration-like process, i.e. fines are captured in the pores of the fibre network. Future improvements of our model to account for fibres species interaction, i.e., the generation of stress between long and short fibres, could potentially predict also this behavior.

Despite simplifications, our model, fitted to literature data (Cotas et al. 2015, 2017), captures and predicts the formation of a gap between the fibre network and the wall, as observed in our experiment (Fig. 1), sufficiently well. For that we are confident to apply our modelling strategy for the design of hydrodynamic fractionation devises in particular and to pulp suspension flow within the laminar plug flow regime in general.

\section{Conclusion}

Streaming fibre networks were known to undergo regime change upon changing the Reynolds number: for low fluid stress, the network is compressed to the channel centre and for high fluid stress, the network is dispersed and solid material is distributed in the suspension. In our current contribution, we documented the relative mobility of fibre-fines and their distribution in the suspension.

Specifically, we probed the streaming suspension near the wall at increasing wall-normal distances and differing flow situations. We found that the quantity of fibre-fines in the gap between the fibre network and the wall, which is essentially free of fibres, is reduced compared to a homogenous fibre-fines concentration. Further, we found the plug interface enriched with fibre-fines what suggests a caging effect during network consolidation, i.e. fibre-fines are transported with the network and their motion relative to long fibres is hindered. This observation is essential for future developments of fractionation devices, since understanding the transport of fibre-fines in these devices is the key to improve energy efficiency and fractionation quality.

Next, we probed fibre suspensions from softwood and hardwood mixtures at different flow regimes. We compared the relative change of fibre-fines sampled from the annular plug flow regime to the turbulent flow regime determined by a mass balance (which captures all fines) and from an optical method (captures only fines larger $33 \mu \mathrm{m}$ ). We found it larger for the mass determined case. From that we conclude, that fibrillar fines, undetectable in optical measurements, are less 
retarded by the network and hence appear to have higher mobility. Effectively that also suggests a changing quality of the removed fines fraction depending on the state of the streaming network: relatively more fibrillar fines are expected for a coherent and consolidated plug. In agreement with this is data on the relative fibril area determined from a dedicated microscopic analysis (Mayr et al. 2017a). As to the different contributions of those fines to a cellulose paper (Mayr et al. 2017b) we suggest to revisit the fibre-fines quality sampled from streaming networks in a future study.

We then formulated a model in the Eulerian frame to compute the migration of fibres and fibre-fines, i.e. consolidation, towards the channel centre. This effort is fueled by our interest to better explain the exact reason for spontaneous fibre segregation and the formation of a wall-near region which is void of long fibres. Our research showed that the elastic properties of a fibre network, as well as fluid turbulence, must be considered to predict the fibre distribution qualitatively correct. The required model closures were adjusted by a comparison with experimental data from Cotas et al. (2015, 2017). Hence, our model parameters were not derived from first principles, and our model is limited to a mono-disperse pulp. Still, we were able to predict the consolidation of a fibre network to the channel centre and its dispersion at higher velocity, i.e., higher fluid stress. The dispersion was however underestimated and the dense fibre network near the channel center was never fully dispersed. Furthermore, we found that our simple model fitted to the suspension flow of fibres qualitatively also captures the suspension behaviour of micro-fibrillated cellulose (Koponen et al. 2019). We note, that future first principle simulations are required to support the closure for fibre migration, elastic flock strength, and turbulence created in the water gap.

Acknowledgments Open access funding provided by Royal Institute of Technology. We acknowledge the support of Angelika Zachl during the development and implementation of the Herschel-Bulkley-type viscosity closure. We thank Thomas Schmid for insightful discussions and proofreading the manuscript. Furthermore, authors gratefully acknowledge the industrial partners Sappi Austria Produktions-GmbH \& Co KG, Zellstoff Pöls AG and Mondi Frantschach GmbH, and the Competence Centers for Excellent Technologies (COMET), promoted by BMVIT, BMDW, Styria and Carinthia and managed by FFG, for their financial support of the K-project
FLIPPR $^{2}$ (Future Lignin and Pulp Processing Research PROCESS INTEGRATION).

Open Access This article is licensed under a Creative Commons Attribution 4.0 International License, which permits use, sharing, adaptation, distribution and reproduction in any medium or format, as long as you give appropriate credit to the original author(s) and the source, provide a link to the Creative Commons licence, and indicate if changes were made. The images or other third party material in this article are included in the article's Creative Commons licence, unless indicated otherwise in a credit line to the material. If material is not included in the article's Creative Commons licence and your intended use is not permitted by statutory regulation or exceeds the permitted use, you will need to obtain permission directly from the copyright holder. To view a copy of this licence, visit http://creativecommons.org/licenses/by/4.0/.

\section{Appendix}

Viscosity model

A rheology model for the fibre-water mixture is implemented in the open-source software OpenFOAM ${ }^{\circledR}$ which consists of a Herschel-Bulkley-type viscosity closure with fibre concentration-dependent parameters. This viscosity model's implementation is in its spirit identical to that proposed by Derakhshandeh et al. (2010). Our implementation basis on the existing Herschel-Bulkley model implemented in OpenFOAM ${ }^{\circledR} 5 . \mathrm{x}^{*}$ The parameters used in this model are summarized in Table S1 of the Supplementary Material. Important to note is that second-order spatial discretization schemes were adopted, while a firstorder accurate temporal scheme with an automatically adjusted time step size was used. Specifically, the time step was chosen based on a maximum Courant number of 0.8 , as well as a maximum permissible viscous time step $\Delta t_{\text {visc }}=0.25 \Delta x /\left(v+v_{t}\right)$ (here $\Delta x$ is the grid spacing, $v_{t}$ the suspension kinematic viscosity, and $v_{t}$ the turbulent kinematic viscosity). This automatic time-step adjustment was key to guarantee stable simulations, also in case of extreme gradients in the suspension viscosity.

\section{Turbulence model}

We tested all low-Reynolds turbulence models available in OpenFOAM ${ }^{\circledR}$ 5.x (for the model implementation and model parameters see github.com/ 
OpenFOAM), and identified the 'kkLOmega' as the most successful to predict a velocity profile as reported by Cotas et al. $(2015,2017)$.

Purely dynamic fibre network stress closure

We assume that fibre migration is caused by an extra normal stress that develops in the sheared fibre network. This stress is in addition to the shear stress already captured by the viscosity model.

Specifically, we model the fibre extra normal stress as a simple extra pressure that is given by Switzer and Klingenberg (2003), as well as Zirnsak et al. (1994):

$\tau_{\text {extra }}=\mathbf{I} \gamma^{n_{S R}} c^{n_{c}} \eta K_{\text {extra }}$.

Here $n_{S R}, n_{c}$ and $K_{\text {extra }}$ are parameters that need to be specified, $\mathbf{I}$ is the identity tensor, $\dot{\gamma}$ is the suspension's local shear rate, $c$ is the fibre consistency, and $\eta$ is the suspension's dynamic viscosity. Consequently, a volumetric fluid-particle interaction force can be calculated from this extra stress simply by:

$\mathbf{f}_{f p}=\nabla \cdot \tau_{\text {extra }}=\eta K_{\text {extra }} \nabla\left(\dot{\gamma}^{n_{S R}} c^{n_{c}}\right)$

We now assume that this fluid-particle force balances the fluid-particle drag force, the latter of which we assume to be of the form

$\mathbf{f}_{\text {drag }}=-n_{f} \mathbf{u}_{\text {drift }} \eta d_{\text {fibre }} K_{\text {drag }}$

Here $K_{\text {drag }}$ is a drag coefficient that needs to be specified, $d_{\text {fibre }}$ is the fibre diameter, and $\mathbf{u}_{\text {drift }}$ is the fluid-particle relative velocity that is needed to close the fibre transport equation. Furthermore, $n_{f}$ is the number of fibres per unit volume, which is related to the fibre consistency via:

$n_{f}=\frac{c \rho_{m i x}}{m_{f}}$

Here $m_{f}$ is the mass of a single fibre, and $\rho_{m i x}$ is the mixture density. The evaluation of the force equilibrium (i.e., fluid-particle drag versus the extra fluidparticle force) leads us to

$\mathbf{u}_{d r i f t}=-\frac{1}{c} \nabla\left(\dot{\gamma}^{n_{S R}} c^{n_{c}}\right) \frac{m_{f}}{\rho_{\text {mix }} d_{\text {fibre }}} K_{\text {drift }}$

For cylindrical fibres the term $A_{f}=\frac{m_{f}}{\rho_{\text {mix }} d_{\text {fibre }}}$ simplifies to $A_{f}=d_{\text {fibre }} L_{\text {fibre }} \frac{\pi}{4} \frac{\rho_{f}}{\rho_{\text {mix }}}$, and hence can be interpreted as a typical projection area of a fibre.
The above equation (i.e., Eq. 10) may diverge for low fibre fractions. Hence, we heuristically introduce the exponent $n_{c, \text { drag }}$ also in the denominator of the model (i.e., we assume a non-linearity also in the drag calculation).

In summary, we model the migration speed of fibres with

$\mathbf{u}_{\text {drift }}=-\frac{1}{c^{n_{c, d r a g}}} \nabla\left(\dot{\gamma}^{n_{S R}} c^{n_{c}}\right) A_{f} K_{d r i f t}$

Hence, four parameters of this migration model must be specified (when lumping $A_{f}$ and $K_{\text {drift }}$ to a single parameter that we denote as $K$ ), and only one of them depends on the projection area of a fibre.

Dynamic and elastic fibre network stress closure

The forming fibre network develops certain elastic properties [this elasticity originates from the stiffness of fibres and frictional contacts. That this is not a kinetic stress, or a stress induced by attractive fibrefibre forces as explained by Schmid and Klingenberg (2000)]. Hence, there must be a contribution to the extra stress that does not depend on the shear rate. In what follows a simple phenomenological model for such an elastic stress is proposed, assuming that this elastic extra stress purely depends on the fibre concentration. Thus, the total extra stress is modelled as:

$\tau_{\text {extra }}=\mathbf{I}\left(\dot{\gamma}^{n_{S R}} c^{n_{c}} \eta K_{\text {extra }}+c^{n_{c, E}} E_{f}\right)$,

Here $n_{c, E}$ is a dimensionless parameter that expresses the sensitivity of elastic forces to the fibre concentration, and $E_{f}$ is a network elasticity constant with units $\left[\mathrm{N} / \mathrm{m}^{2}\right]$. This extra stress model leads us to the following expression for the drift flux:

$\mathbf{u}_{d r i f t}=-\frac{1}{c^{n_{c, d r a g}}} \nabla\left(\dot{\gamma}^{n_{S R}} c^{n_{c}}+c^{n_{c, E}} K_{e l}\right) A_{f} K_{d r i f t}$

here $K_{e l}$ is a dimensionless elastic stress contribution.

Fibre transport equation

The spatio-temporal evolution of the local fibre concentration, i.e., the fibre transport equation, is

$\partial_{t} c+\nabla \cdot\left(\left[\mathbf{u}+\mathbf{u}_{\text {drift }} c\right]\right)=\nabla \cdot\left(D_{f} \nabla c\right)$ 
Here $\mathbf{u}$ is the suspension velocity, and $D_{f}$ is a fibre diffusivity. The latter is considered for numerical reasons only, i.e., to stabilize the simulation (specifically, we have used a fixed ratio of fibre diffusivity and kinematic suspension viscosity of $1 / 100$ that leads to stabilization but is small enough to not affect the concentration profiles). The above equation states that fibres are transported by a drift between fibres and liquid in addition to a bulk (i.e., suspension) motion. Also, fibres can be dispersed due to the fibre diffusivity $D_{f}$, and may accumulate in a volume element leading to a temporal change of the local fibre concentration.

\section{References}

Athley K, Granlöf L, Söderberg D et al (2012) Mechanical retention-Influence of filler floc size and grammage of the fibre web. Nord Pulp Pap Res J 27:202-207. https://doi. org/10.3183/npprj-2012-27-02-p202-207

Bergström R (2005) Fibre flow mechanisms. KTH Royal Institute of Technology

Brennan D (2001) The numerical simulation of two-phase flows in settling tanks. Imperial College of Science, Technology and Medicine

Celzard A, Fierro V, Kerekes R (2009) Flocculation of cellulose fibres: new comparison of crowding factor with percolation and effective-medium theories. Cellulose 16:983-987. https://doi.org/10.1007/s10570-009-9314-0

Cotas C, Silva R, Garcia F et al (2015) Application of different low-Reynolds $\mathrm{k}-\varepsilon$ turbulence models to model the flow of concentrated pulp suspensions in pipes. Procedia Eng 102:1326-1335. https://doi.org/10.1016/j.proeng.2015.01. 263

Cotas C, Branco B, Asendrych D et al (2017) Experimental study and computational fluid dynamics modeling of pulp suspensions flow in a Pipe. J Fluids Eng. https://doi.org/10. 1115/1.4036165

Derakhshandeh B, Hatzikiriakos SG, Bennington CPJ (2010) Rheology of pulp suspensions using ultrasonic Doppler velocimetry. Rheol Acta 49:1127-1140. https://doi.org/10. 1007/s00397-010-0485-2

Derakhshandeh B, Kerekes RJ, Hatzikiriakos SG, Bennington CPJ (2011) Rheology of pulp fibre suspensions: a critical review. Chem Eng Sci 66:3460-3470. https://doi.org/10. 1016/j.ces.2011.04.017

Duffy GG (2006) Measurements, mechanisms and models: some important insights into the mechanisms of flow of fibre suspensions. Annu Trans Nord Rheol Soc 14:19-31

Duffy GG (2007) The significance of mechanistic-based models in fibre suspension flow. Nord Pulp Pap Res J 18:074-080. https://doi.org/10.3183/npprj-2003-18-01-p074-080

Haavisto S, Cardona MJ, Salmela J et al (2017) Experimental investigation of the flow dynamics and rheology of complex fluids in pipe flow by hybrid multi-scale velocimetry.
Exp Fluids 58:158. https://doi.org/10.1007/s00348-0172440-9

Hammarström D (2004) A model for simulation of fiber suspension flows. Licent thesis, R Inst Technol

Hemström G, Moller K, Norman B (1976) Boundary layer studies in pulp suspension flow. TAPPI J 59:115-118

Hyll K (2015) Size and shape characterization of fines and fillers-a review. Nord Pulp Pap Res J 30:466-487. https:// doi.org/10.3183/NPPRJ-2015-30-03-p466-487

Jäsberg A, Kataja M (2009) Flow regimes in flows of wood fibre suspensions. In: 14th Pulp Pap. Fundam. Res. Symp, pp 161-180

Karnis A (1997) Pulp fractionation by fibre characteristics. Pap Ja Puu 79:480-488

Kerekes RJ (2006) Rheology of fibre suspensions in papermaking: an overview of recent research. Nord Pulp Pap Res J 21:598-612. https://doi.org/10.3183/npprj-2006-21-05p598-612

Kerekes RJ, Schell CJ (1992) Characterization of fibre flocculation regimes by a crowding factor. J Pulp Pap Sci 18:32-38

Kerekes RJ, Schell CJ (1995) Effects of fiber length and coarseness on pulp flocculation. TAPPI J 78:133-139

Koponen A, Haavisto S, Salmela J, Kataja M (2019) Slip flow and wall depletion layer of microfibrillated cellulose suspensions in a pipe flow. Annu Trans Nord Soc Rheol 27:13-20

Krochak PJ, Olson JA, Martinez DM (2009) Fiber suspension flow in a tapered channel: the effect of flow/fiber coupling. Int J Multiph Flow 35:676-688. https://doi.org/10.1016/j. ijmultiphaseflow.2009.03.005

Krochak PJ, Olson JA, Martinez DM (2010) Near-wall estimates of the concentration and orientation distribution of a semi-dilute rigid fibre suspension in Poiseuille flow. J Fluid Mech 653:431-462. https://doi.org/10.1017/ S0022112010000406

Lindström SB, Uesaka T (2008) Particle-level simulation of forming of the fiber network in papermaking. Int J Eng Sci 46:858-876. https://doi.org/10.1016/j.ijengsci.2008.03. 008

MacKenzie J, Söderberg D, Swerin A, Lundell F (2018) Turbulent stress measurements of fibre suspensions in a straight pipe. Phys Fluids 30:025104. https://doi.org/10. 1063/1.5008395

Marchioli C, Fantoni M, Soldati A (2010) Orientation, distribution, and deposition of elongated, inertial fibers in turbulent channel flow. Phys Fluids 22:1-14. https://doi.org/ 10.1063/1.3328874

Martinez DM, Buckley K, Lindström A, et al (2001) Characterizing the mobility of papermaking fibres during sedimentation. Sci Papermak 12th Fundam Res Symp 16:225-254

Mayr M, Eckhart R, Sumerskiy I et al (2015) Flippr $^{\circ}$ - an industrial research project in Austria. Tappi J 14:209-212

Mayr M, Eckhart R, Bauer W (2017a) Improved microscopy method for morphological characterisation of pulp fines. Nord Pulp Pap Res J 32:244-252. https://doi.org/10.3183/ NPPRJ-2017-32-02-p244-252

Mayr M, Thaller A, Eckhart R, Bauer W (2017b) Characterization of fines quality and their independent effect on sheet 
properties. In: Transactions of the 16th fundamental research symposium. Oxford, pp 299-322

Meyer H (1964) An analytical treatment of the laminar flow of annulus forming fibrous suspensions in vertical pipes. TAPPI J 47:78-84

Nikbakht A, Madani A, Olson JA, Martinez DM (2014) Fibre suspensions in Hagen-Poiseuille flow: transition from laminar plug flow to turbulence. J Nonnewton Fluid Mech 212:28-35. https://doi.org/10.1016/j.jnnfm.2014.08.006

Norman B, Moller K, Ek R, Duffy GG (1977) Hydrodynamics of papermaking fibres in water suspensions. BPBIF 6th Fund. Res. Symp. Fibre-Water Interact. Papermak. $195-250$

Olson JA (2001) Fibre length fractionation caused by pulp screening, slotted screen plates. J Pulp Pap Sci 27:255-261

Parker JD (1972) The sheet-forming process. The Technicl Association of the Pulp and Paper Industry, Atlanta, USA

Paulapuro H (2007) Papermaking science and technology book 8: papermaking part 1. Stock Preparation and Wet End, Paperi ja Puu Oy

Redlinger-Pohn JD, Jagiello LA, Bauer W, Radl S (2016) Mechanistic understanding of size-based fiber separation in coiled tubes. Int J Multiph Flow. https://doi.org/10.1016/j. ijmultiphaseflow.2016.04.008

Redlinger-Pohn JD, Bauer W, Radl S (2017a) Fractionation of fibre pulp in a hydrodynamic fractionation device: influence of reynolds number and accept flow rate. Trans 16Th Fundam Res Symp Held Oxford Sept 2017 209-228

Redlinger-Pohn JD, König J, Radl S (2017b) Length-selective separation of cellulose fibres by hydrodynamic fractionation. Chem Eng Res Des. https://doi.org/10.1016/j.cherd. 2017.08.001

Sampson W (2001) The structural characterisation of fibre networks in papermaking processes-a review. Sci Papermak Trans XIIth Fundam Res Symp Bak CF 1205-1288
Sandgren B, Wahren D (1960) Studies on Pulp Crill. Part 2. A Crill Screen Sven Papperstidning 63:854-858

Schmid CF, Klingenberg DJ (2000) Mechanical flocculation in flowing fiber suspensions. Phys Rev Lett 84:290-293. https://doi.org/10.1103/PhysRevLett.84.290

Schmid CF, Switzer LH, Klingenberg DJ (2000) Simulations of fiber flocculation: effects of fiber properties and interfiber friction. J Rheol (N Y N Y) 44:781-809. https://doi.org/10. $1122 / 1.551116$

Schmid T, Redlinger-Pohn JD, Radl S (2019) Length-based hydrodynamic fractionation of highly networked fibers in a mini-channel. Nord Pulp Pap Res J 34:182-199. https:// doi.org/10.1515/npprj-2018-0086

Soszynski R (1987) The formation and properties of coherent flocs in fibre suspensions. University of British Columbia

Steenberg B, Wahren D (1960) Concentration gradients in boundary layers of streaming fibre suspensions. Sven Papperstidning 63:347-355

Steenberg B, Sandgren B, Wahren D (1960) Studies on pulp crill. Part 1. Suspended fibrils in paper pulp fines. Sven Papperstidning 63:395-397

Switzer LH, Klingenberg DJ (2003) Rheology of sheared flexible fiber suspensions via fiber-level simulations. J Rheol (N Y N Y) 47:759-778. https://doi.org/10.1122/1.1566034

Switzer LH, Klingenberg DJ (2004) Flocculation in simulations of sheared fiber suspensions. Int J Multiph Flow 30:67-87. https://doi.org/10.1016/j.ijmultiphaseflow.2003.10.005

Zirnsak MA, Hur DU, Boger DV (1994) Normal stresses in fibre suspensions. J Nonnewton Fluid Mech 54:153-193. https:// doi.org/10.1016/0377-0257(94)80020-0

Publisher's Note Springer Nature remains neutral with regard to jurisdictional claims in published maps and institutional affiliations. 